\title{
Implications of sleep quality and eating behavior in obesity prevention: A cross-sectional study in young adults.
}

\author{
$\underline{\text { Maria Fernanda Zeron-Rugerio }}^{1,2}$, Alina Schieren ${ }^{1}$, Antoni Diez-Noguera ${ }^{3}$, Trinitat Cambras ${ }^{3}$ \\ and Maria Izquierdo-Pulido ${ }^{4,2}$ \\ ${ }^{1}$ Department of Nutrition, Food Science and Gastronomy. School of Pharmacy and Food Science. University of \\ Barcelona., Barcelona, Spain, \\ ${ }^{2}$ Institut de Recerca en Nutrició i Seguretat Alimentària (INSA-UB) University of Barcelona., Barcelona, Spain, \\ ${ }^{3}$ Department of Biochemistry and Physiology. School of Pharmacy and Food Science. University of Barcelona, \\ Barcelona, Spain and \\ ${ }^{4}$ Department of Nutrition, Food Science and Gastronomy. School of Pharmacy and Food Science. University of \\ Barcelona, Barcelona, Spain
}

\begin{abstract}
Introduction

Food intake behavior is implicated in many chronic health issues, including obesity. Thus, understanding the underlying factors that affect food intake is important. Several studies have associated sleep restriction to increased dietary intake and poor food choices, which can be driven by hedonic mechanisms, specially food palatability. Given that people eat for different reasons other than stress, including boredom, anxiety, sadness or joy, our aim was to study the potential association between sleep quality and the cognitivebehavioral nature of food intake (eating behaviors, EB), as well as their impact on body mass index in a population of young adults.

\section{Materials and Methods}

One thousand twenty eight young adults (78\% females; $21.4 \pm 4$.1years) were included in a cross-sectional study. The following questionnaires were used: Pittsburg Sleep Quality Index to assess sleep quality (scores $>5$ indicate poor sleep quality), the Three Factor Eating Questionnaire to assess the three dimensions of EB (emotional, uncontrolled and restraint eating) (Higher scores indicate higher EB). Additionally, height, weight, body mass index (BMI) and the level of physical activity were evaluated. Statistical analysis were performed using SPSS v24, and adjusted for confounding variables.
\end{abstract}

\section{Results}

Sixty one percent of the studied population had a poor sleep quality, whereas no significant associations were found between poor sleep quality, gender or age. Poor sleep quality was associated with uncontrolled $(p<0.001)$ and emotional ( $p<0.001)$ eating, as well as with BMI $(\mathrm{p}<0.001)$. In addition, emotional eating was associated to BMI $(\mathrm{p}<0.001)$. Finally, linear regression model showed that BMI is associated with poor sleep quality $(\mathrm{p}<0.01)$ and emotional eating $(\mathrm{p}<0.001)$.

\section{Discussion}

Our findings suggest that poor sleep quality is frequent among young adults. Furthermore, poor sleep quality is associated with higher emotional and uncontrolled eating, showing that poor sleep might be associated with a higher tendency to over eat when coping to emotionally negative emotions (sadness, anger, depression, anxiety), and a high tendency to lose control over food intake itself. Finally, the association between poor sleep quality and high emotional eating can have a negative impact over BMI, even in young adults. Hence, interventions designed to improve weight status would benefit from focusing on sleep quality in order to reduce emotional and uncontrolled eating.

\section{Conflict of Interest}

There is no conflict of interest 